GAPP, número 24, noviembre de 2020

Sección: RECENSIONES

Recibido: 15-07-2020

Modificado: 02-09-2020

Aceptado: 28-09-2020

DOI: https://doi.org/10.24965/gapp.i24.10828

Páginas: 143-146

\title{
LÓPEZ, Silvia y PLATERo, Lucas R. (eds.): Cuerpos marcados. Vidas que cuentan y políticas públicas
}

\section{LóPEZ, Silvia \& PlateRo, Lucas R. (eds.): Cuerpos marcados. Vidas que cuentan y políticas públicas}

\author{
Cristina Herranz \\ Universidad Autónoma de Madrid (España) \\ ORCID: https://orcid.org/0000-0003-3763-9281 \\ cristina.herranz@uam.es
}

\section{NOTA BIOGRÁFICA}

Graduada en Ciencia Política y de la Administración Pública (2015), y Máster en Democracia y Gobierno (2016) por la Universidad Autónoma de Madrid (UAM). Realiza su doctorado y es profesora en el departamento de Ciencia Política y Relaciones Internacionales (UAM). Su investigación se centra en la transformación digital y la innovación democrática y la interseccionalidad y los estudios de género.

\section{RESUMEN}

Recensión del libro de LóPEZ, Silvia y PLATERo, Lucas R. (eds): Cuerpos marcados. Vidas que cuentan y políticas públicas. Barcelona: Bellaterra, 2019, 260 págs.

\section{PALABRAS CLAVE}

Políticas públicas; género; feminismo; vidas precarias.

\begin{abstract}
Review of the book from LóPEZ, Silvia y PLATERo, Lucas R. (eds): Cuerpos marcados. Vidas que cuentan y políticas públicas. Barcelona: Bellaterra, 2019, 260 pp.
\end{abstract}

\section{KEYWORDS}

Public policies, gender, feminism, precarious life.

Cuerpos marcados, Vidas que cuentan y políticas públicas se constituye como una de las primeras obras colectivas en español cuya propuesta fundamental es la de situar los cuerpos como centro de la discusión y del análisis de políticas públicas. Silvia López y R. Lucas Platero coordinan este volumen, cuyo diagnóstico muestra cómo los cuerpos son situados en posiciones de jerarquía y vulnerabilidad.

El trabajo cuenta con aportaciones de casi una veintena de autores y autoras de procedencia académica y/o activista muy diversa que, analizando los discursos y estrategias institucionales, ofrecen una visión multidisciplinar de los efectos que producen estos discursos en las condiciones de vida de las personas. Los autores y autoras, desde la propia experiencia de sus cuerpos marcados, introducen una serie de temáticas de gran relevancia para el feminismo contemporáneo y que pueden servir a su vez como guía de acción política. En sus propias palabras: «Nos sumamos, por tanto, a las críticas feministas que denuncian que 
estamos hablando del cuerpo sin el cuerpo, sin tener en cuenta tampoco que la perspectiva más frecuente sobre el cuerpo es aquella que lo presenta como algo neutral» (pág. 13)

Este enfoque plantea entender la acción/ inacción institucional como un elemento capaz de discriminar entre los cuerpos (re)produciendo desigualdades y vulnerabilidades. Con ello, se busca centrar la atención en qué vidas merecen la pena ser vividas, qué cuerpos se cuidan y cuáles son aquellos que reciben violencia o discriminación sistémica. Introducir la idea de los «cuerpos marcados» como categoría, invita al lector a reflexionar acerca de los cuerpos no normativos frente a la idea de la neutralidad de los cuerpos, del cuerpo promedio o normal para el que se desarrollan la mayoría de las políticas públicas, no contando con la disidencia, con la no normalidad. Con ello, se muestra cómo los binarismos pueden ser foco primordial de desigualdades y se defiende la existencia de la múltiple expresión y diferencia de los cuerpos. La expresión «cuerpos marcados» ofrece así un paraguas conceptual que explora los márgenes, o cómo determinados cuerpos son señalados como el cuerpo no modelo. En este sentido, la ausencia de marca proporciona una situación de privilegio promoviendo una dualidad que proyecta la imagen de «los cuerpos» (la normalidad) frente a «los otros cuerpos» (pág. 14).

Esta reflexión parte de debates teóricos propuestos con anterioridad tales como la «precariedad de la vida» de Judith Butler (2006, 2010), la biopolítica de Michel Foucault (1979), o las posibilidades de vida o de muerte que se describen en el concepto de la necropolítica (Mbembé, 2011), entre otros. Desde el punto de partida de sus antecedentes intelectuales, la obra busca responder a las siguientes cuestiones de actualidad: ¿qué estrategias se utilizan en las diferentes áreas de políticas públicas para proteger o privilegiar a determinados cuerpos? ¿Cuáles son las categorías no cuestionadas dentro de la acción pública? ¿Qué ejes o qué desigualdades se deben tener en cuenta para comprender la experiencia de estos cuerpos marcados? ¿Qué políticas pueden disponer de un carácter transformador?

Para abordar estas cuestiones, el libro se articula en cuatro partes diferenciadas por sus temáticas y enfoques. Mientras que las dos primeras partes del libro responden a trabajos de investigación a través de metodologías diversas, las dos últimas se componen de textos de carácter más reflexivo cuyo propósito es aportar material para la discusión, ofreciendo nuevas preguntas para el futuro del feminismo académico y activista. Por ello, el orden de lectura no tiene por qué ser riguroso.

A continuación, se comentan las principales contribuciones de cada uno de estos bloques, poniendo de relieve la importancia y originalidad del enfoque utilizado en la obra en relación a las políticas públicas y su análisis y estudio.

El primero de los bloques "Una aproximación teórica: el cuerpo en el centro" recoge el marco teórico base sobre el que se asientan los presupuestos normativos y antecedentes teóricos de la obra y que se compone de dos capítulos. En el primero de ellos, Elvira Burgos presenta un profundo debate filosófico que desgrana las principales aportaciones de la teoría de Judith Butler en relación a la importancia del cuerpo y la performatividad del género. El segundo capítulo de este bloque es un artículo colectivo (Marisela Montenegro Martínez, Lucía Egaña Rojas y Joan Pujol Tarrés) que ofrece un relato histórico acerca de cómo se ha ido configurando el concepto queer entrelazándose, en este punto, el desarrollo del activismo y el pensamiento feminista. La apropiación del insulto, la propuesta de «de-construcción» de los cuerpos o prácticas sociales - las estrategias de recomprensión de lo normal son algunas de las reflexiones que recoge este análisis, reflejando así la mezcla entre teoría y activismo existente en lo queer, con especial atención a su desarrollo en España y ofreciendo una visión de lo que denominan cuerpos de(s)generados.

El segundo bloque, el más extenso de la obra, titulado: Cuerpos marcados y políticas públicas incluye cuatro capítulos sobre distintos ejemplos que ilustran cómo las políticas públicas pueden generar situaciones de mayor desigualdad o vulnerabilidad en cuerpos ya de por sí precarios. En este bloque se encuentra el capítulo de Pablo Santoro, realizando un análisis discursivo acerca de las políticas sobre el VIH desde la década de los ochenta hasta la actualidad. En él se trata específicamente el área de la salud como espacio de distribución desigual de vulnerabilidad y violencia y se muestra cómo las estrategias sobre salud tienen efecto directo sobre los cuerpos. A través de estas políticas y su genealogía, el autor pone de relieve tres lógicas que se reproducen en el ámbito sanitario y que marcan los cuerpos precarizándolos: exclusión, normalización y medicalización (pág. 90).

En el segundo capítulo de este bloque, Alba Artiaga plantea un debate acerca de los cuidados y la Ley de Dependencia (Ley 39/2006 de Promoción de la Autonomía Personal y Atención a personas en situación de dependencia) a través del análisis de las políticas públicas y su implementación: para ello, se centra en cómo la manera en la que se define o nombra el problema puede ocasionar la comprensión del fenómeno como un problema individual y favorecer una forma concreta de resolverlo. Las cadenas 
globales de cuidado, el tipo de trabajo feminizado o la mercantilización de los cuidados son algunos de los asuntos que la autora estudia mostrando que la cultura en torno a los cuidados, el deber cuidar, distribuye responsabilidades y marca los cuerpos de manera diferencial. Este análisis normativo jurídico se sustenta además sobre más de sesenta entrevistas en profundidad para comprender el fenómeno, incluyendo a actores clave que participaron en el diseño y desarrollo de la política y a sujetos implicados con diferentes perfiles.

El tercer capítulo del bloque lo firman Melania Moscoso y Lucas Platero acuñando el término de cripwashing (lavado de cara) y abordando las políticas sobre el derecho a decidir sobre el propio cuerpo (la reforma de la ley $2 / 2010$ sobre salud sexual y reproductiva y de interrupción voluntaria del embarazo). Recibe el nombre de cripwashing «el uso interesado que se hace del discurso de las personas con discapacidad para justificar la conculcación de derechos de otros colectivos en situación de desventaja, en este caso, el derecho al aborto de las mujeres en el estado español» (págs. 135-136). Con este capítulo y a través del análisis de los discursos en el debate sobre la reforma del aborto (utilizando el enfoque de Carol Bacchi (2009)), muestran la instrumentalización de los derechos de las personas con discapacidad en el debate mediático sobre el aborto en España que buscaba enfrentar discursivamente los derechos de las personas con diversidad funcional y las mujeres.

Este bloque finaliza con la aportación de Daniel J. García López que, desde una perspectiva jurídica, se centra en las violencias que pueden generar los procesos de cambio de nombre y sexo en el Registro Civil sobre las personas trans, particularmente en los menores de edad (Ley 3/2007). Tras realizar un análisis de la jurisprudencia, muestra cómo el sistema jurídico español marca a los cuerpos que no se ajustan a la norma. El autor pone de manifiesto que los requisitos para que se produzca el cambio registral producen diversas exclusiones: por nacionalidad, por minoría de edad y por disponer o no de una «capacidad suficiente». Entre otras cuestiones que señala el autor, para que pueda realizarse el proceso, el diagnóstico médico o psicológico debe dictaminar disforia de género y asegurar que el solicitante no tiene otro trastorno (pág. 165). Con ello, se le conceden a la medicina y los expertos poder sobre las vidas.

El tercer bloque del libro titulado "Piezas para el debate" se compone de textos más breves que ofrecen material para la discusión, abriendo nuevas puertas para el debate y poniendo en el centro a los cuerpos. Se encuentran en esta sección los textos de Carolina León Almeyda, María Rodó, Nuria Sadurní, Bárbara Tardón, Amparo Cano y Yayo Herrero, por orden de aparición en la obra. En este bloque se discuten la maternidad, la idea de familia y lo que implica para la mujer, los permisos, el alquiler de vientres, la violencia obstétrica, el empleo y la precariedad, el derecho a la reproducción asistida, la necropolítica queer y las tensiones que puede producir la ley catalana 11/2014, la violencia sexual, la descripción e invisibilización del cuerpo en el ámbito de la salud mental y la perspectiva ecofeminista de comprensión de los cuerpos.

Por último, el cuarto bloque que cierra el libro recoge una conversación de las editoras con Esther (Mayoko) Ortega Arjonilla en el que se reflexiona acerca de la experiencia de escritura de Cuerpos Marcados, cuáles fueron sus motivaciones. En esta conversación final se abordan los problemas o marcas derivadas de la racialización pero, además, se vuelven a remarcar las preguntas que configuran el leitmotiv del libro: «¿qué cuenta como una vida?» o «¿desde dónde pueden nacer estrategias para sobrevivir o para resistir?» (pág. 243).

Uno de los aportes más relevantes de esta obra radica en que cada uno de los capítulos ofrece, de una manera u otra, una imagen de la práctica de la interseccionalidad en el estudio y análisis de políticas públicas, enfoque todavía poco habitual en la literatura en español. Desde un punto de vista normativo, este enfoque adopta la perspectiva foucaultiana/ butleriana de inclusión de los cuerpos en todo análisis y en la interpretación de la distribución del poder, al tiempo que alienta al lector a buscar una comprensión más holística de fenómenos de exclusión y precarización de las vidas. Desde un punto de vista práctico, situar el foco de análisis en la experiencia de vida permite ofrecer una imagen de las desigualdades que confluyen en diversos sectores sociales vulnerables. Por ello, los capítulos de orientación académica se complementan con una perspectiva que busca incitar a la acción y a la reflexión. Así, más allá de tratar de ofrecer un mapa o una descripción exhaustiva de las vulnerabilidades en España, propone abrir interrogantes, cuestionar la neutralidad de la acción institucional y visibilizar que determinadas dinámicas sociales e institucionales dificultan la sostenibilidad y calidad de la vida. El estilo de la obra, que puede resultar poco homogéneo, sirve también como ejemplo de diferentes formas de aproximación a la problemática que se presenta. El lector encontrará ejemplos de análisis de políticas, referencias históricas, pero también relatos de vida, lo que hace que esta obra comulgue con diferentes tipos de literatura. Por todo ello, Cuerpos Marcados ofrece una serie 
GAPP.NuevaÉpoca - N. ${ }^{\circ} 24$, noviembre 2020 - ISSN:1989-8991 - DOI:https://doi.org/10.24965/gapp.i24.10828 - [Págs. 143-146]

LÓPEZ, Silvia y PLATERO, Lucas R. (eds.): Cuerpos marcados. Vidas que cuentan y políticas públicas

Cristina Herranz

de aportaciones que pueden resultar de gran interés para un público amplio y particularmente para personas interesadas en el análisis de políticas públicas desde una perspectiva de género.

\section{REFERENCIAS BIBLIOGRÁFICAS}

BACCHI, C. (2009): Policy analysis: What is the problem represented to be. French Forest (Sydney): Pearson.

BUTLER, J. (2006): Vida precaria. El poder del duelo y la violencia. Buenos Aires: Paidós.

BUTLER, J. (2010): Marcos de guerra: las vidas lloradas. Barcelona, etc.: Paidós.

FOUCAULT, M. (2009): Nacimiento de la biopolítica: curso del Collège de France (1978-1979). Tres Cantos (Madrid): Akal.

MBEMBE, A. (2011): Necropolítica. Sobre el gobierno privado indirecto. Barcelona: Melusina. 